\title{
CGRP-Antikörper reduziert Migränetage
}

Fragestellung: Ist der gegen den CGRP-Rezeptor gerichtete Antikörper AMG 334 zur Prophylaxe der episodischen Migräne wirksam?

Hintergrund: Calcitonin gene-related peptide (CGRP) ist ein wichtiger Botenstoff in der Pathophysiologie der Migräne. Es wird während Migräneattacken ausgeschüttet. Die parenterale Gabe von Sumatriptan reduziert die CGRP-Spiegel dramatisch und in zeitlicher Assoziation mit der Besserung der Migränesymptome. Daher wurden humanisierte Antikörper gegen das Peptid selbst oder den CGRP-Rezeptor entwickelt, mit der Intention, sie als Prophylaktika einzusetzen. Für eine Reihe der CGRPAntagonisten liegen bereits die positiven Daten von Phase-IIStudien vor. AMG 334 ist ein humanisierter monoklonaler Antikörper, der hochpotent und selektiv an den CGRP-Rezeptor bindet. Die Halbwertszeit beträgt 21 Tage. Der Antikörper muss subkutan einmal im Monat injiziert werden. Aufgrund seiner Größe, ist es ihm nicht möglich, die Bluthirnschranke zu überwinden und zentrale CGRP-Rezeptoren anzugreifen.

Patienten und Methodik: Es handelt sich um eine multizentrische randomisierte placebokontrollierte Doppelblindstudie, in die Migränepatienten im Alter zwischen 18 und 60 Jahren mit vier bis 14 Migränetagen pro Monat eingeschlossen wurden. In dieser Dosisfindungsstudie wurde AMG 334 in den Do-

Sun H, Dodick DW, Silberstein $S$ et al. Safety and efficacy of AMG 334 for prevention of episodic migraine: a randomised, double-blind, placebo-controlled, phase 2 trial. Lancet Neurol 2016; 15: 382-90 sierungen $7 \mathrm{mg}, 21 \mathrm{mg}$ und $70 \mathrm{mg}$ mit Placebo verglichen. Der primäre Endpunkt war die Änderung der Migränetage pro Monat zwischen einer Baseline von vier Wochen und der zwölfwö- chigen doppelblinden Behandlungsphase. Außerdem wurden Nebenwirkungen erfasst. Nach der randomisierten Studie konnten die Patienten in eine offene Studie wechseln.

Ergebnisse: Insgesamt wurden 483 Patienten in die Studie eingeschlossen. 160 Patienten erhielten Placebo, jeweils 108 Patienten $7 \mathrm{mg}$ und $21 \mathrm{mg}$ sowie 107 Patienten $70 \mathrm{mg}$ AMG 334. Der Antikörper wurde alle vier Wochen subkutan injiziert. Die Patienten waren im Mittel 40 Jahre alt. $82 \%$ waren Frauen. Die Migräne bestand im Mittel seit 20 Jahren, die durchschnittliche Zahl der Migränetage pro Monat betrug 8,7, die Zahl der Migräneattacken 5,4 .

Unter Placebo betrug die Reduktion der Migränetage pro Monat 2,3. Für die 7-mg-Dosis von AMG 334 wurde eine Reduktion von 2,2 Tagen und für 21-mg-Dosis von 2,4 Tagen gezeigt. Für beide Dosierungen war der Unterschied zu Placebo nicht signifkant. Die 70-mg-Dosis war signifikant wirksamer und führte zu einer Reduktion um 3,4 Migränetage pro Monat ( $\mathrm{p}=$ 0,021). Die 50\%-Responderrate betrug 30\% für Placebo, $29 \%$ für $7 \mathrm{mg}, 34 \%$ für $21 \mathrm{mg}$ und $46 \%$ für $70 \mathrm{mg}$ AMG 334. Auch dieser Unterschied war für die hohe Dosis signifikant.

AMG 334 wurde sehr gut vertragen. Es gab keine schwerwiegenden unerwünschten Arzneimittelwirkungen, die mit der Behandlung assoziiert waren. Alle anderen Nebenwirkungen waren in der Placebogruppe gleich häufig wie in den drei Verumgruppen. Neun der 316 Patienten (2\%) entwickelten neutralisierende Antikörper. Bei den Laboruntersuchungen ergaben sich keine Veränderungen.

Schlussfolgerung: Die Ergebnisse rechtfertigen den Einsatz von AMG 334 in einer Dosis von $70 \mathrm{mg}$ alle vier Wochen in einer größeren Phase-III-Studie zur Prophylaxe der episodischen Migräne.

\section{- Kommentar von Hans-Christoph Diener, Essen}

\section{Vergleichbare Wirksamkeit wie Betablocker und Antiepileptika}

Die Ergebnisse des Antikörpers der Firma Amgen sind vergleichbar mit den Ergebnissen der Phase-II-Studien der CGRPAntikörper von Lilly, Teva und Alder. Die Antikörper selbst unterscheiden sich. Bei den Substanzen von Lilly, Teva und Alder handelt es sich um Antikörper gegen das CGRP-Molekül, bei der Firma Amgen um einen Antikörper, der an den CGRP-Rezeptor bindet. Offenbar hat dies aber weder Konsequenzen für die Wirksamkeit, noch für Nebenwirkungen. Besonders hervorzuheben ist das gute Nebenwirkungsprofil der CGRP-Antikörper. Die Wirksamkeit ist in etwa vergleichbar mit der Wirksamkeit von Betablockern und Antiepileptika. Sofern die neuen CGRPAntikörper in den Phase-III-Studien ihre Wirksamkeit belegen, sind sie am ehesten geeignet für Patienten, bei denen die derzeitige prophylaktische medikamentöse Therapie nicht wirkt, nicht toleriert wird oder kontraindiziert ist.

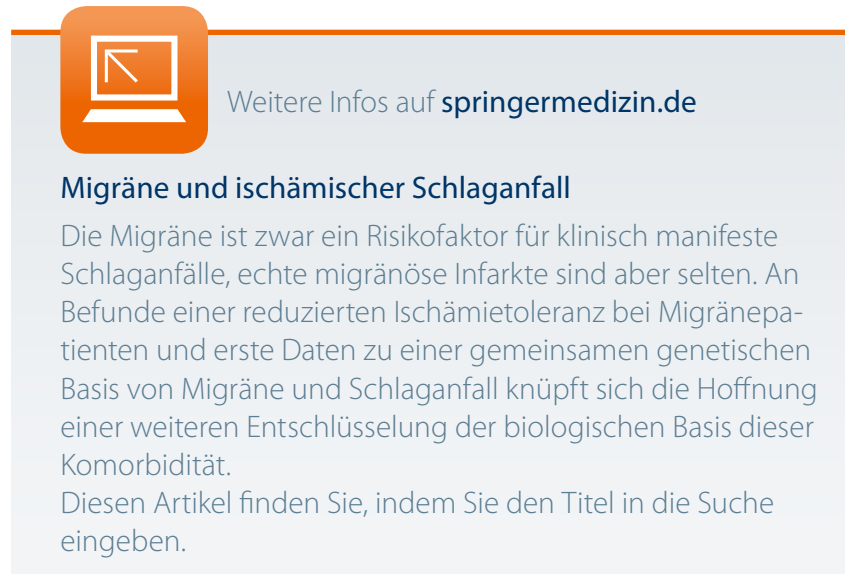

